\section{MS12-P8 Applying robust statistical methods to assess the effect of excess solvent in long wavelength data collection}

Sam Horrell ${ }^{1}$, Ramona Duman ${ }^{1}$, Juan Sanchez-Weatherby ${ }^{1}$, Richard Strange ${ }^{2}$, Armin Wagner ${ }^{1}$

1. Diamond Light Source, Harwell Science and Innovation Campus, Chilton, Didcot, Oxfordshire, OX11 ODE, UK

2. Molecualr Biophysics Group, School of Biological Sciences, University of Liverpool, Crown Street, Liverpool, Merseyside, L69 $7 \mathrm{ZB}, \mathrm{UK}$

email: sam.horrell@liv.ac.uk

As modern macromolecular crystallography (MX) is addressing increasingly challenging projects, there is a greater need to improve the quality of diffraction data. Amongst the most limiting factors affecting data quality are crystal quality, radiation damage and the signal-to-noise ratio. This study focuses on improving the signal-to-noise-ratio by removing surrounding solvent to reduce background scatter and absorption. Although not considered critical for standard MX experiments, in the light of long-wavelength and microcrystal MX, it becomes a limiting factor. This is evident from the variety of sample handling and mounting systems developed to reduce these effects; including the loopless mounting method (Kitago et al. 2010), the use of adhesive materials for harvesting (Kitatani et al. 2008), coating samples with atomically thin graphene sheets (Wierman et al. 2013), shaping macromolecular crystals with PULSA (Kitano et al. 2005) and excising crystals grown on ultrathin films with a femtosecond laser (Marquez \& Cipriani. 2014).

Here (Horrell et al. 2015) we use robust statistical analysis to show manipulation of macromolecular crystals and removal of excess solvent in a humid air stream can be done at no cost to crystal integrity. To test this, we transferred large populations of macromolecular crystals from nylon loops to Kapton MicroMounts, using micromanipulators for precise movement, and a humidity control device (Sanchez-Weatherby et al. 2009), to preserve crystals during the process. Diffraction data collected at $\lambda=1 \AA$ from lysozyme, thaumatin, insulin and ferritin show no significant difference between test and control samples, emphasising this methods potential. Similar tests with data collected at longer wavelengths $(\leq 2 \AA)$ on protein crystals from $P$. aeruginosa, $P$. caratovorum and $P$. syringae have revealed protein dependent effects on data quality based on exposure to the humid air stream and data collection around $\lambda=2 \AA$.

Horrell, S. et al., (2015). To be Submitted to Acta crystallographica. Section D

Kitago, Y. et al., (2010). Journal of Applied Crystallography 43, 341-346

Kitano, H. et al., (2005). Journal of bioscience and bioengineering 100, 50-53

Kitatani, T. et al., (2008). Applied Physics Express 1, $1-3$

Sanchez-Weatherby, J. et al., (2009). Acta crystallographica D65, 1237-1246

Wierman, J. L. et al., (2013). J Appl Crystallogr 46, 1501-1507

Marquez, J. A. \& Cipriani, F. (2014). Methods in molecular biology 1091, 197-203

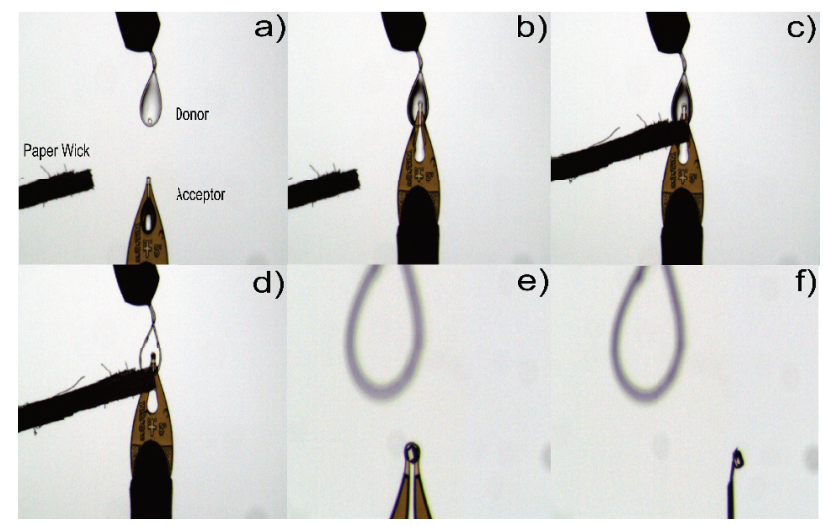

Figure 1. A microscope view of the step wise transfer (a-f) of a 50 um insulin crystal from a nylon CryoLoop to a Kapton MicroMount in a humidity controlled stream by removing excess solvent.

Keywords: Humidity Control, Sample Handling, Long Wavelength X-ray Crystallography, Data Quality. 